Revue des patrimoines

$2 \mid 2002$

La monographie d'architecture

\title{
L'apport de l'archéologie du bâti dans la monographie d'architecture
}

\section{Nicolas Reveyron}

\section{(2) OpenEdition}

1 Journals

Édition électronique

URL : http://journals.openedition.org/insitu/1200

DOI : $10.4000 /$ insitu. 1200

ISSN : 1630-7305

Éditeur

Ministère de la Culture

Référence électronique

Nicolas Reveyron, «L'apport de l'archéologie du bâti dans la monographie d'architecture », In Situ [En ligne], 2 | 2002, mis en ligne le 23 avril 2012, consulté le 10 décembre 2020. URL : http:// journals.openedition.org/insitu/1200; DOI : https://doi.org/10.4000/insitu.1200

Ce document a été généré automatiquement le 10 décembre 2020.

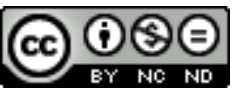

In Situ Revues des patrimoines est mis à disposition selon les termes de la licence Creative Commons Attribution - Pas d'Utilisation Commerciale - Pas de Modification 4.0 International. 


\title{
L'apport de l'archéologie du bâti dans la monographie d'architecture
}

\author{
Nicolas Reveyron
}

\section{Introduction}

1 L'histoire de l'art affiche dans son intitulé même les deux lieux qui organisent sa recherche ${ }^{1}: l^{\prime}$ histoire, d'une part, c'est-à-dire une méthodologie et un travail spécifiques sur les textes et les sources archivistiques; d'autre part, l'art en tant que création matérielle, objet d'une étude archéologique, au sens où l'entendait l'enseignement supérieur au XIX ${ }^{\mathrm{e}}$ siècle : dans sa leçon d'ouverture du cours d'antiquités grecques et latines de la faculté de Lyon (année 1877), Gustave Bloch précise que «L'archéologie étudie les formes diverses que l'homme a su donner à la matière pour la faire servir à ses besoins ou à ses plaisirs... L'archéologie n'est donc pas seulement l'histoire de l'art, bien qu'elle ait aussi ce caractère et même qu'elle l'ait eu longtemps d'une manière exclusive » 2.

2 Aujourd'hui, l'étude matérielle de la production artistique, pour paraphraser le titre d'un colloque célèbre ${ }^{3}$, bénéficie de moyens d'investigation très développés, sophistiqués et en plus grand nombre. Elle intéresse l'art dans ses objets et dans le savoir-faire des praticiens. En ce qui concerne les tableaux, la statuaire, les objets d'art, les manuscrits etc., l'apport des laboratoires spécialisés est considérable et souvent décisif dans la connaissance intime des œuvres, mais aussi des artistes (histoire matérielle des pièces, repentirs, savoir-faire, «manières ».... $)^{4}$. L'histoire de l'architecture inscrit sa démarche dans la même complémentarité duale: comme l'affirmait déjà Pérignon en 1841, les monuments « sont de précieux témoins à interroger. La mémoire ne leur manque jamais ; ils n'ont pas d'intérêt à mentir et peut-être y a-t-il plus de foi à accorder à l'histoire écrite en architecture qu'à toute autre $»^{5}$. 


\section{Dialogue du texte et de l'édifice}

3 Très tôt, d'éminents spécialistes ont évoqué les difficultés présentées par ce dialogue nécessaire. Dès 1911, dans sa préface au Recueil de textes relatifs à l'histoire de l'architecture et à la condition des architectes en France au moyen-âge, Victor Mortet soulignait ce décalage qui interdit la superposition exacte des archives et des faits matériels, et, par voie de conséquence, relativise la convergence entre la documentation écrite et les données de l'analyse monumentale ${ }^{6}$. Huit ans plus tard, Camille Enlart explicitait les termes de la problématique et l'explorait dans pratiquement toute son étendue :

4 «Enfin, lorsque l'on a sainement apprécié la portée d'un texte, il reste à en faire une application juste, et cela est parfois plus difficile encore.

5 Ainsi, Suger estimait que Dagobert avait bâti Saint-Denis ; cependant, l'édifice avait été rebâti depuis, sous le roi Pépin. Ce genre d'erreur est resté fréquent, et bien des archéologues sont plus excusables que l'abbé de Saint-Denis, car il arrive souvent qu'une première construction est racontée par des textes, tandis que la mention des reconstructions postérieures est perdue ou omise, soit par négligence, soit pour donner des illusions sur l'ancienneté de l'édifice. Aussi est-il nécessaire de confronter les monuments avec les textes : un texte attribuait au $\mathrm{XI}^{\mathrm{e}}$ siècle la chapelle Sainte-Croix de Montmajour; M. Brutails, le trouvant inconciliable avec le caractère de l'architecture, eut l'idée de le soumettre à une étude critique, et découvrit qu'il s'appliquait à une chapelle voisine.

6 Par contre, Quicherat, dans une remarquable étude sur Saint-Gilles, utilise tous les textes, mais ayant négligé d'examiner à fond l'édifice, il se trompe sur leur portée : il prend pour des refaçons du XIII ${ }^{e}$ siècle celles du XVI ${ }^{e}$ ou du XVII ${ }^{e}$ siècle, faute d'avoir visité la travée aujourd'hui séparée et formant sacristie, qui, seule, a échappé à ces dernières restaurations $»^{7}$.

7 Les contradictions entre texte et monument trouvent leurs sources dans l'une et l'autre approche: les imprécisions, inhérentes à toute description médiévale ${ }^{8}$, établie sans le recul nécessaire à un travail historique et, plus évidemment encore, à des documents juridiques, comptables, législatifs, administratifs ou autres dont la finalité n'est pas une description architecturale ou archéologique, mais la consignation de faits sélectionnés sur critères et spécifiques entrant exactement dans le champ qui leur est propre $^{9}$; les erreurs, involontaires ou intentionnelles ${ }^{10}$; les lacunes, délicates à déceler ; les première impressions trompeuses contre lesquelles, dès 1900, J. A. Brutails mettait les archéologues en garde, les invitant à ne pas céder au leurre des apparences en traduisant « vétusté » ou " maladresse " par "ancienneté $»^{11}$ les critiques d'authenticité menées sur le terrain, souvent malaisées à établir formellement; la perte des archives ou la disparition des bâtiments... À ces causes s'ajoutent les questions de langage, spécialisés, techniques, corporatistes, codés... Tout particulièrement, les problèmes de traduction, qui sont de deux ordres. D'une part, la difficulté de traduire des termes médiévaux ${ }^{12}$ ou dialectaux, souvent rédhibitoire concernant le champ lexical des termes de métiers ${ }^{13}$. D'autre part, la distanciation culturelle, susceptible de superposer un filtre de lecture déformant sur la réalité référentielle, ou même d'en interdire l'intelligence; notons d'ailleurs que cette difficulté est à l'origine d'une floraison de dictionnaires archéologiques, à l'usage des littéraires comme des historiens de l'art. Il s'en est suivi une imprécision du vocabulaire couramment employé, que seul pouvait régler un dictionnaire réalisé par des spécialistes 
de différents domaines comme le Principes d'analyse scientifique, architecture, méthode et vocabulaire ${ }^{14}$.

Figure 1

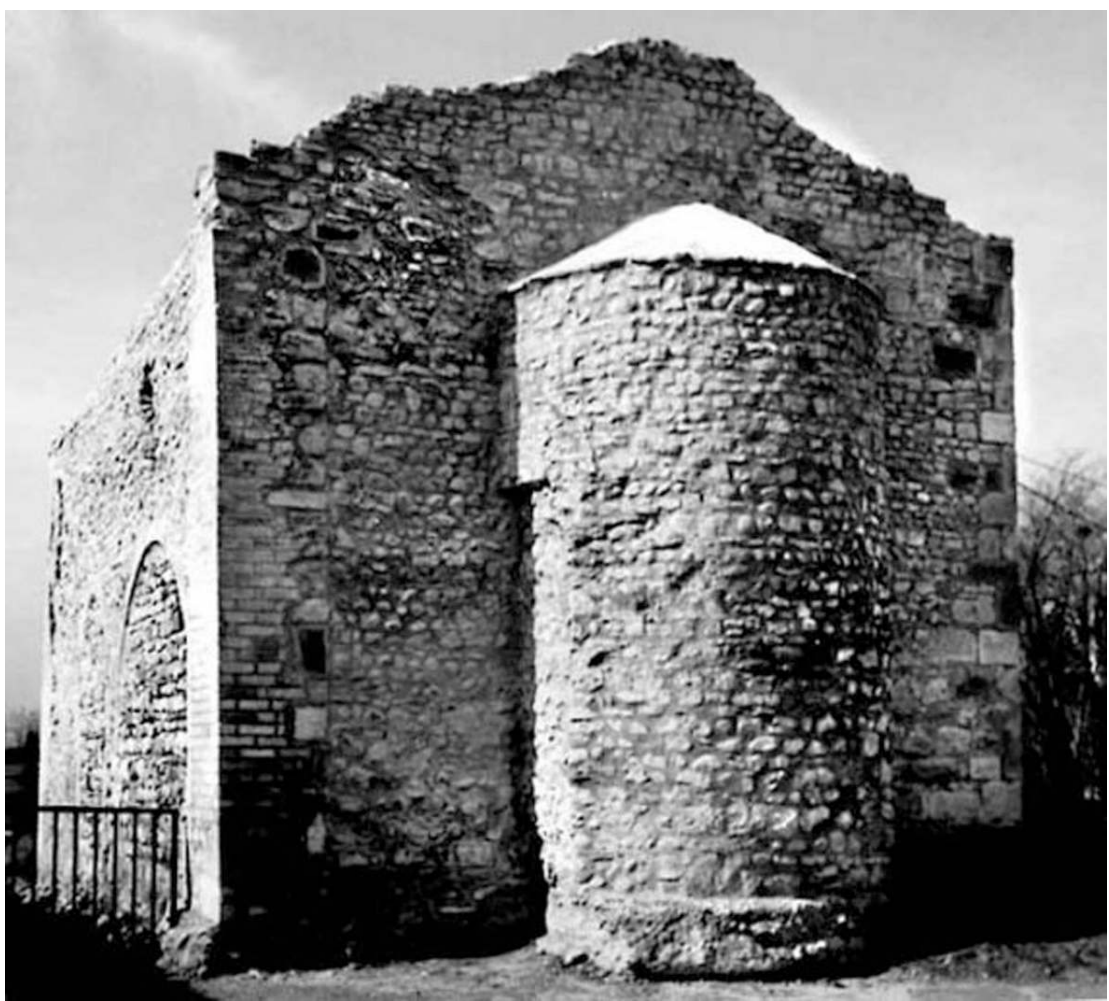

Saint-Didier-de-Formans (01), chapelle du XVIIe siècle. Cette chapelle, appuyée sur le mur goutterot nord d'une église du XIe siècle, aujourd'hui disparue, a remplacé une installation primitive du XIVe siècle et subi des modifications jusqu'au XIXe siècle. Dans un contexte de documentation écrite extrêmement lacunaire, l'analyse archéologique du bâti a permis de retrouver les traces du chantier roman, des premières chapelles et des différentes phases d'aménagement

(c) N. Reveyron

Toutefois, les historiens de l'art n'ont jamais abandonné les observations du bâti. Dans son manuel, J.-A. Brutails codifiait ainsi cette nécessité : «Une première précaution à prendre consiste à réunir tous les éléments d'information, à envisager le problème sous toutes ses faces, dans les documents et les monuments. Il faut s'enquérir des édifices, en même temps qu'on les observe eux-mêmes $»^{15}$. Mais, quoique fines et pertinentes, ces dernières ont été le plus souvent réduites au rôle d'auxiliaire de l'histoire de l'art, utiles pour confirmer les chronologies proposées : on vérifiait dans les maçonneries les traces des événements révélés par les textes et l'on organisait la lecture de l'édifice en prenant pour guide la trame du discours historique. Cette orientation a longtemps interdit une théorisation et une systématisation de la démarche archéologique; partant, elle a considérablement retardé l'élaboration d'instruments de recherche spécifiques, capables de fonder un discours scientifique autonome. Pour prendre un exemple concret, on considère souvent, aujourd'hui encore, le changement d'appareil dans un même édifice comme un indice chronologique: ni la notion de techniques de construction, ni celle d'esthétique du bâti ne sont réellement prises en compte.

Dans ce dialogue sans symétrie, l'aporie majeure surgit au moment où l'on tente d'appliquer les données révélées dans un domaine de recherche à celles de l'autre; elle 
naît du décalage plus ou moins marqué dont parlait Victor Mortet. Telle substruction estelle l'église signalée au $\mathrm{X}^{\mathrm{e}}$ siècle, une salle d'apparat de l'Antiquité ou un bâtiment vernaculaire non documenté ? Tel pilier offert par ce chanoine dans le troisième quart du XII ${ }^{\mathrm{e}}$ siècle est-il cette pile sommée d'un chapiteau à crochet primitif ou ce support que décorent des chapiteaux romans tardifs? L'hésitation et l'incertitude consécutive trouvent leur source dans la primauté donnée à une démarche par rapport à l'autre : il s'avère tout aussi vain de chercher à calquer un texte sur un ensemble de murs que de vouloir imposer les parties d'un bâtiment à un ensemble de données textuelles. Des expériences les plus récentes ${ }^{16}$, il faut tirer cette leçon méthodologique: dans chaque domaine, l'analyse doit conserver son autonomie et être menée à bien dans son seul cadre et avec ses seuls instruments ; c'est au second temps de la démarche qu'appartient la mise en regard des résultats, au moment où les connaissances historiques et les analyses archéologiques sont déjà pleinement fondées, au moment où elles ont trouvé leur justification dans leur cohérence interne, et non dans l'appui de données extérieures au champ d'investigation.

\section{L'archéologie du bâti}

En 1993, Les Nouvelles de l'archéologie ont consacré un dossier à «L'archéologie du bâti médiéval urbain $»^{17}$. C. Arlaud et J. Burnouf y posaient la question : «L'archéologie du bâti existe-t-elle ? ${ }^{18}$. Une réponse contradictoire a été apportée à cette question par les Journées d'études organisées du 20 au 23 septembre 1994 à Pont-à-Mousson par l'École du Patrimoine et consacrées à l'Archéologie du bâti ${ }^{19}$; la même année, P. Garmy faisait le point dans un article sur l' "Etat de la recherche française en archéologie du bâti médiéval urbain. Eléments pour un bilan 1986-1993 $»^{20}$. Comme toute science en gestation, pour laquelle ni la méthodologie, ni l'objet de la recherche n'apparaissent encore clairement dans leur spécificité, cette pratique archéologique a reçu des noms divers : archéologie $d u$ bâti, archéologie du bâti urbain, archéologie du bâti médiéval urbain, archéologie du mur, archéologie du construit, ce que la recherche italienne appelle archeologia dell'architettura ${ }^{21}$ ou archeologia del costruito ${ }^{22}$; il faut y ajouter l'archéologie d'élévation et l'archéologie d'élévation monumentale ${ }^{23}$. Inflation verbale? Dérive scientifique? Non. Il n'existe qu'une seule archéologie, "praxis» définie dans ses trois modalités par Michel de Boüard: archéométrie, analyse quantitative des phénomènes étudiés; archéographie, étude par observation et description, y compris graphiques ; archéologie, réflexion menée sur les observations réalisées, suivant une méthodologie et des outils conceptuels spécifiques. 
Figure 2

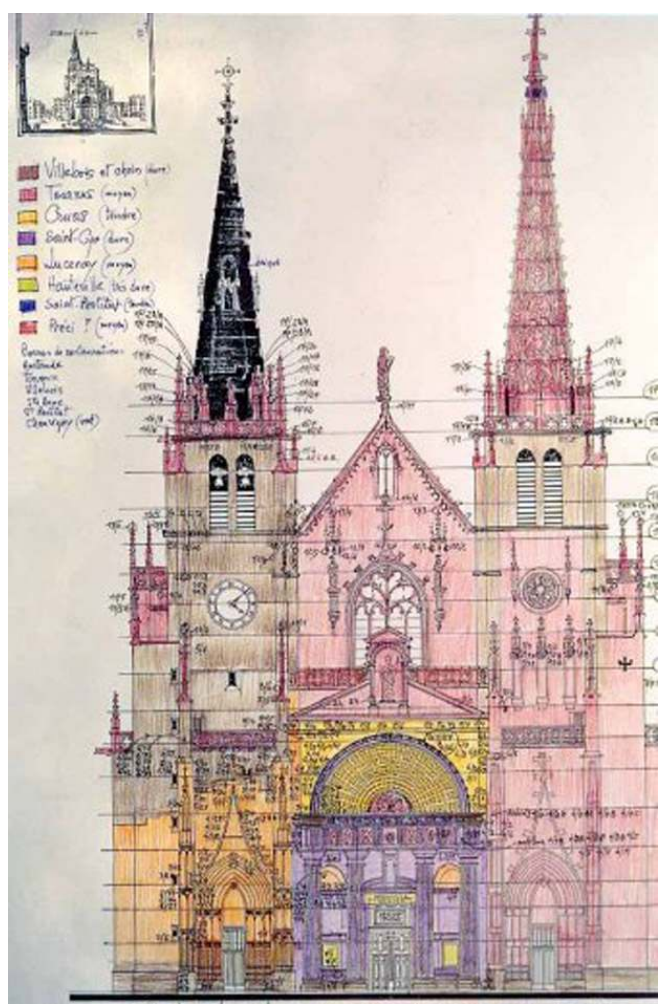

Saint-Didier-de-Formans (01), chapelle du XVIIe siècle. Relevé et mise en couleur du mur sud de la chapelle (mur goutterot nord de l'église), par Nolwen Fravalo

11 Cette forme d'archéologie s'est développée dans le mouvement des restaurations de monuments historiques, notamment par le biais des études préalables mises en place progressivement depuis deux décennies. C'est grâce à une étroite collaboration, dans le cadre des Directions régionales des Affaires culturelles, avec les Conservations régionales des Monuments Historiques et les Services régionaux de l'Archéologie que la recherche dans ce domaine s'est développée et a confirmé ses intuitions. La recherche archéologique a bénéficié, sur le terrain, d'un partage de savoir avec les acteurs de la restauration : architectes, sculpteurs, tailleurs de pierre, maçons, échafaudeurs, et alii. Ces travaux ont permis en retour la constitution d'une documentation hors pair, parce que réalisée «le nez sur la pierre » et avant les interventions nécessairement destructrices d'indices ${ }^{24}$.

12 Mais il est certain que, si la méthode archéologique reste une, l'objet en oriente très sensiblement l'application et donne à la démarche adoptée son allure propre. Les édifices religieux, plus précisément les lieux de culte, n'ont pas à subir de profondes modifications, si l'on excepte les accidents graves ou les changements brutaux d'affectation: l'organisation de l'espace intérieur et la distribution des masses répond à des exigences ou des intentions qui débordent les modes. À l'inverse, comme l'a écrit Deshoulières, les constructions de défense ont connu des évolutions souvent radicales: «Beaucoup ont péri ou ont été transformés en ruines et sont méconnaissables; d'autres ont reçu des améliorations nécessitées par le progrès des moyens d'attaque $»^{25}$.

L'habitat a été soumis a des transformations encore plus fréquentes, du fait de l'évolution des modes et des mœurs : pour ne prendre qu'un exemple, le passage du groupe familial, 
associant les générations et les collatéraux, à la famille mononucléaire, moins hiérarchisée et plus soucieuse d'intimité, a entraîné de profondes transformations dans les volumes internes et les circulations (autonomie des pièces, migration interne des escaliers, création de couloirs et portes...). Dans ces conditions, il existe une spécificité de l'archéologie des élévations monumentales, par référence à l'architecture dite vernaculaire, mais il est évident que cette spécificité ne se traduit ni en terme de particularité, ni, surtout, de spécialité : principes et méthodologie sont indivisibles.

\section{La pratique archéologique}

Pratiquer l'archéologie du bâti, c'est appliquer les méthodes de l'archéologie du sous-sol à des édifices en élévation ${ }^{26}$, en dépassant ce seuil psychologique que Catherine Arlaud et Joëlle Burnouf ont appelé «la frontière du bitume »: comme l'affirmait Pierre Garmy dans son introduction aux Journées d'études de Pont-à-Mousson, « il y a une archéologie du mur, comme il y a une archéologie de la couche ».

En effet, dans une construction, qui suscite parfois aussi des investigations lourdes ${ }^{27}$, comme sous le sol, l'étude et la fouille ${ }^{28}$ reposent sur la même démarche archéologique : les méthodes de relevés graphiques et de mise au net sont les mêmes ${ }^{29} ;$ l'analyse des stratifications, horizontales ou verticales (par exemple, la superposition des enduits), le décryptage des perturbations (baies creusées, passages bouchés...), l'établissement des chronologies relatives relèvent des mêmes processus, depuis l'enregistrement des données jusqu'à la constitution des diagrammes et le traitement graphique des phasages ; les éventuelles datations absolues utilisent aussi des poutres, du charbon de bois, des vestiges végétaux, la terre cuite (brique, carreau...), etc. Seuls les champs de recherche peuvent présenter des spécificités: différentes formes de «ruptures $»^{30}$, repères de la chronologie relative; vestiges laissés par les échafaudages, en rapport notamment avec le phasage du chantier ${ }^{31}$; traces laissées par les instruments de taille de pierre, dont l'évolution détermine quelques repères chronologiques ${ }^{32}$; marques lapidaires ${ }^{33}$, depuis les marques comptables (gestion de la production de pierre et vérification des livraisons) ou les marques techniques (marques de pose, d'assemblage, de hauteur d'assise...), jusqu'aux marques de tailleurs de pierre, qui peuvent parfois s'interpréter comme une forme primitive de signature ${ }^{34}$; modules des blocs, qui orientent la recherche dans le domaine technique, économique ou typochronologique ${ }^{35}$, voire l'épaisseur des joints ${ }^{36}$. 


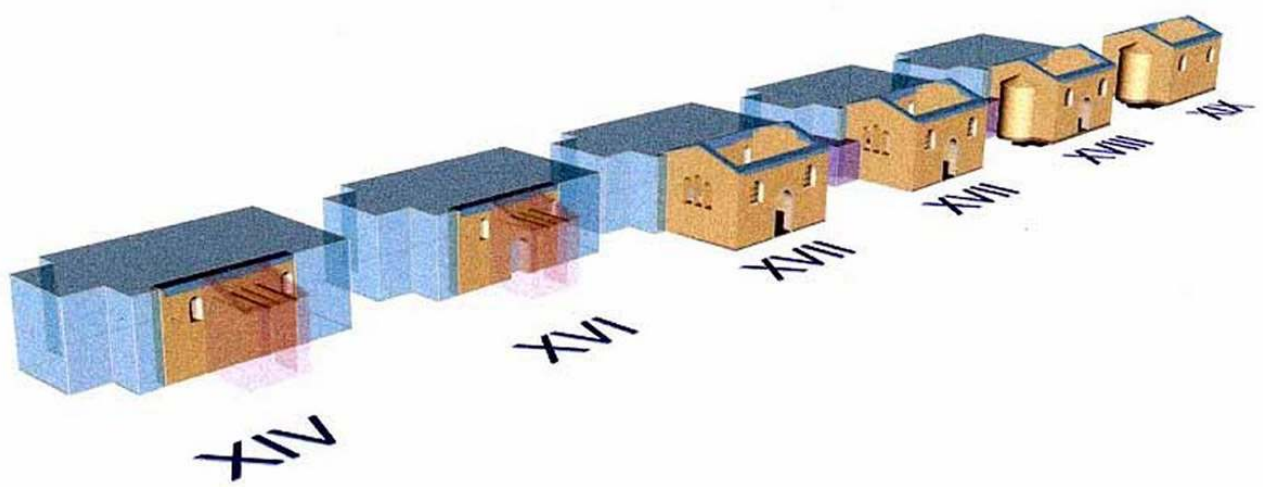

Saint-Didier-de-Formans (01), chapelle du XVIIe siècle. Restitution 3 D des chapelles successives et des différents aménagements, du XIVe au XIXe siècle (l'église a été restituée par un volume bleu, d'après les données du plan cadastral), par Baptiste Reveyron

L'histoire de l'édifice se dessine donc dans son bâti, depuis l'ouverture du chantier et le creusement des fondations, jusqu'aux aménagements intérieurs ${ }^{37}$. L'implantation de l'édifice, la présence de vestiges anciens dans le sous-sol et l'organisation du chantier en fonction du vieil édifice encore debout ou de bâtiments extérieurs contraignants sont discernables dans le désaxement de supports, le manque d'intégration des espaces, le tracé non rectiligne des murs ${ }^{38}$, les désordres dans les maçonneries, voire des cas plus atypiques comme, à la cathédrale de Lausanne, les stigmates du franchissement de la nef par la rue principale de la ville haute ${ }^{39}$. Dans le même ordre d'idée, on repère parfois dans le parement intérieur (par exemple, les cathédrales de Bourges ${ }^{40}$, de Vienne et, sans doute, de Lyon $^{41}$ ) les traces d'une clôture du chantier ${ }^{42}:$ l'apparat des cérémonies exigeait une stricte séparation de l'espace liturgique et du chantier qui l'entourait; la clôture rétablissait une sorte d'unité monumentale provisoire ${ }^{43}$. D'un autre côté, des contraintes ponctuelles, comme la préservation d'espaces de circulation ${ }^{44}$ ou des besoins liturgiques, ont aussi marqué parfois le bâti : à la cathédrale de Lyon, l'analyse des échafaudages médiévaux a mis en évidence un phasage des travaux corroboré par les impératifs de la liturgie lyonnaise.

\section{Ouvertures et collaborations}

17 L'ouverture à toutes les problématiques suscitées par l'objet étudié s'est imposée progressivement aux chercheurs. Car il est évident que l'objet oriente son étude. Dans le cas de l'habitat urbain, par exemple, l'archéologie du bâti est indissociable de l'étude historique du site : mise en place initiale en rapport avec le parcellaire ; agrandissements, réguliers ou non, des bâtiments en fonction de la rue et de la forme de la parcelle (par exemple, occupation de la partie profonde du terrain, puis de la cour); réunion de plusieurs parcelles; recul de la façade à l'occasion d'un alignement; ajouts divers et constructions adventices; modifications continuelles de la circulation, des espaces et des passages, parfois même des niveaux d'occupation; exhaussement du sol transformant le rez-de-chaussée en cave et la cave en second sous-sol, etc. 
L'analyse du bâti débouche alors sur des considérations plus larges qui intéressent principalement l'histoire de la ville, mais aussi l'histoire matérielle et culturelle, notamment les dimensions juridiques ou sociales du bâtiment ${ }^{45}$. L'affirmation du statut social, par exemple, se lit dans le rapport de l'habitat avec la rue (la «belle façade », le plus souvent en pierre de taille) et dans les aménagements intérieurs, notamment la grande salle : dans les maisons de style roman du sillon rhodanien et de la Provence, la belle façade de pierre de taille est le plus souvent réservée à la rue principale; dans le cas de maisons formant l'angle d'une rue, la façade latérale est montée en moellons, sauf les rares exceptions où elle est rendue bien visible par un large dégagement ${ }^{46}$. Le prestige du site importe moins que celui du commanditaire; on rencontre les mêmes tendances dans des castrum comme ceux de la bordure méridionale du Massif Central : «Si à partir du XII e siècle, la maison à étage, connaissant une diffusion plus large, n'est plus le signe d'une distinction sociale, l'apparition d'une construction de qualité liée principalement à l'emploi d'une pierre de taille, marque toujours l'existence d'une demeure aristocratique et/ou privilégiée $»^{47}$.

Figure 4

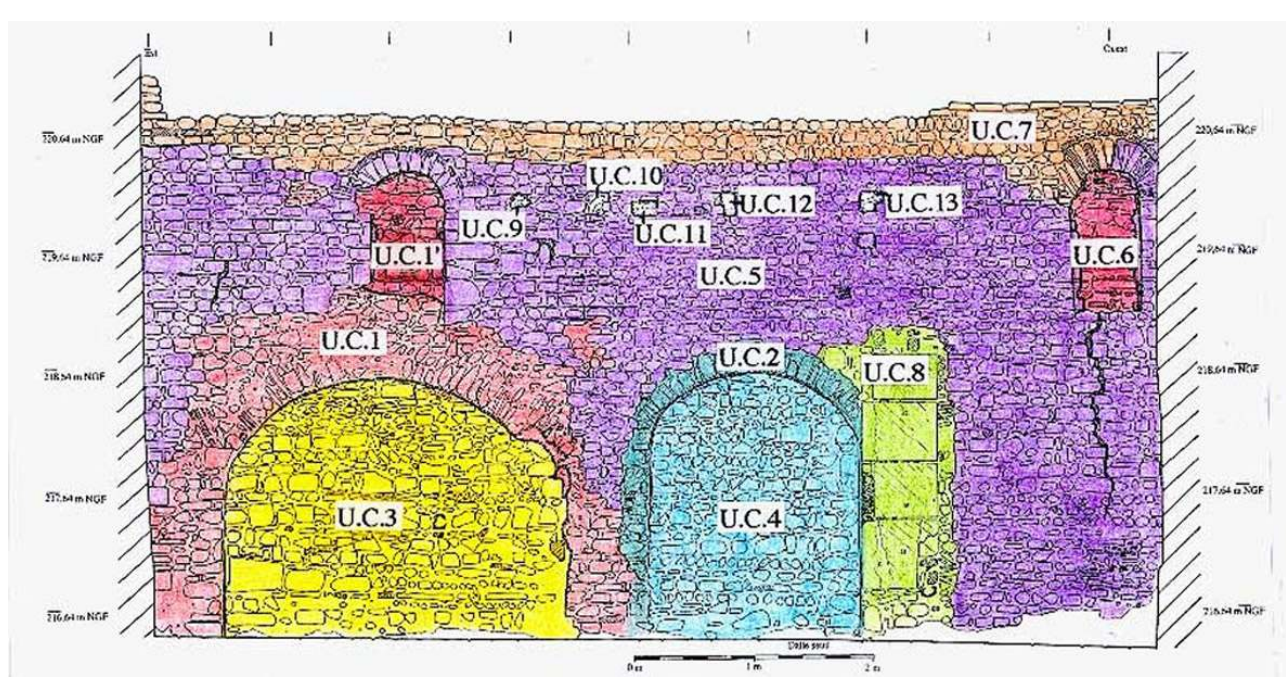

Lyon, ancienne collégiale Saint-Nizier, façade (XVe-XIXe). Localisation des qualités de pierre utilisées dans la façade, par Maximilien Zychla, tailleur de pierre

Ces problématiques n'apparaissent pas dans la seule architecture vernaculaire. À Lyon, les études archéologiques menées sur la cathédrale et l'ancienne collégiale Saint-Nizier ont abouti à des résultats inattendus, mettant en relation des domaines de recherche qu'on imagine rarement associés. À la cathédrale de Lyon, par exemple, dans les premières décennies du XIII ${ }^{e}$ siècle, le chœur s'est fracturé dans sa totalité selon une ligne nord-sud. Cet accident relève de l'histoire politique et juridique de la ville : l'édifice a été reconstruit plus grand par l'archevêque Guichard de Pontigny (1165-1182) qui a choisi de l'allonger vers l'est sur des terrains instables, mais appartenaient de jure à l'archevêque, comte de la ville ${ }^{48}$, plutôt qu'à l'ouest, sur de vastes terrains pourtant fermes et libres ; sans doute la querelle l'opposant aux chanoines, auxquels le pape l'avait imposé comme archevêque, lui a-t-elle interdit cette alternative ${ }^{49}$. Dans un tout autre ordre d'idée, mais toujours à la cathédrale, l'analyse archéologique du bras sud du transept et l'étude conjointe de l'éclairement de l'édifice, effectuée par la Laboratoire d'éclairement de l'École Nationale de Travaux Publics de l'État de Vaulx-en-Velin ${ }^{50}$, ont mis en évidence les 
profondes transformations subies par le transept, lieux d'un renouvellement majeur du premier gothique lyonnais ${ }^{51}$.

Quant à l'ancienne collégiale Saint-Nizier $\left(\mathrm{XIV}^{\mathrm{e}}-\mathrm{XVI} \mathrm{e}^{\mathrm{e}}\right)$, les campagnes menées sur le chevet ont révélé, outre les étapes de l'édification, les techniques de construction ou l'originalité de certaines solutions techniques (comme le mode d'évacuation des eaux pluviales), des pans entiers de l'histoire moderne. L'étude des trous de poutre et des traces d'aménagements à peine lisibles a en effet permis de reconstituer l'histoire matérielle de l'aula des chanoines, ses agrandissements et son lotissement tardif, mais aussi l'organisation d'un curieux aménagement, dont il ne restait pratiquement rien, et qui est sans doute une recluserie.

\section{Conclusion}

On le voit, l'apport de l'archéologie du bâti à la monographie d'architecture est loin d'être négligeable. Il faut insister sur la nécessité de la production d'une importante documentation graphique (les relevés d'élévation et leur mise au net), de très haute qualité et d'une exactitude archéologique. Il faut insister aussi sur l'accès aux détails de la construction qu'autorisent seules les investigations menées à partir des échafaudages. Cette double vision, rapprochée (sur l'échafaudage) et globale (grâce aux relevés au 20e), permet d'obtenir ce « relief » nécessaire à la compréhension générale de l'édifice.

\section{NOTES}

1. Il est bien évident que les documents graphiques (iconographie historique, plans, carnets de croquis, photographies anciennes ... voire graffiti) sont d'un apport considérable, et ils sont intégrés depuis longtemps à la recherche en histoire de l'art. Mais, pour la clarté de l'exposé, le propos doit rester centré sur le rapport texte-archéologie.

2. Leçon d'ouverture citée par Lyne Therrien, L'histoire de l'art en France, genèse d'une discipline universitaire, Paris, Éditions du CTHS, 1998, p. 232-233. Sur le cas spécifique de l'histoire de l'architecture, voir: Simona Talenti, L'histoire de l'architecture en France, Émergence d'une discipline, Paris, Picard, 2000. On sait qu'à son origine, l'enseignement de l'archéologie, directement associé aux musées, s'est donné pour but la connaissance des objets conservés, c'est-à-dire la lecture qu'en autorise la connaissance du contexte culturel, au sens large du terme, dans lequel ils ont été créés.

3. Artistes, artisans et production artistique au Moyen-Age, Colloque international C.N.R.S. Université Rennes II 2-6 mai 1983, dir. X. Barral i Altet, Paris, 1986 (3 vol.).

4. Citons une des premières synthèses, celle de Madeleine Hours : Les secrets des chefs-d'œuvre, Paris, 1964.

5. Déclaration à la chambre, lors de la séance du 4 mars 1841. Cité par P. Léon La vie des monuments français. Destruction, restauration. Paris, 1951, p. 117.

6. Paris, 1911. Réédition du CTHS, Paris, 1995, préface de Léon Pressouyre.

7. Camille Enlart, Manuel d'archéologie française depuis les temps mérovingiens jusqu'à la Renaissance, I - Architecture religieuse, Paris, 1919, p. 109. Pour un exemple récent de prise en 
compte de la spécificité de chaque champ d'investigation et un essai de théorisation, voir: François Comte, «Les piles des moulins du grand pont d'Angers (c. 1440?): apport et contradiction de l'archéologie, des textes et de la dendrochronologie ", La construction en Anjou au Moyen Age, actes de la table ronde d'Angers des 29 et 30 mars 1996, éd. D. Prigent, N.-Y. Tonnerre, Presses de l'université d'Angers, 1998, p. 189-216.

8. Cela est vrai aussi pour les descriptions antiques ou modernes. À notre époque, les descriptions, scientiques ou techniques, utilisent un vocabulaire spécialisé qui donnent une grande précision au texte, mais lui enlève tout effet de visualisation des choses.

9. Luc François Génicot insiste sur l'obscurité introduite dans les descriptions par une recherche stylistique déplacée (descriptions de châteaux près de Calais par Lambert d'Ardres), les passages équivoques (descriptions de Saint-Trond) ou les lacunes (renseignements actés par Galbert de Bruges sur Saint-Donatien à propos du meurtre du comte de Flandre en 1128). Luc François Génicot, L'architecture. Considérations générales. Typologie des sources du Moyen Age occidental, fasc. 29, B-I, A, 1. Turnhout, 1978, p. 53.

10. Pour ne prendre qu'un exemple, la mention fréquente, aux $\mathrm{XI}^{\mathrm{e}}$ et $\mathrm{XII}^{\mathrm{e}}$ siècles, d'églises reconstruites «depuis les fondations" relève souvent plus d'une clause de style à visée panégyrique que d'une réalité archéologiquement avérée.

11. "Les archéologues ne sauraient trop se défendre, en matière de date, contre les impressions irréfléchies : l'appareil barbare de Saint-Martin-du-Canigou, formé de dalles schisteuses à peine taillées, et l'état de ruine de l'édifice sont peut-être pour quelque chose dans l'attribution de cette église au début du XI ${ }^{\mathrm{e}}$ siècle ", J. A. Brutails, L'Archéologie du Moyen Age et ses méthodes, Paris, 1900, p. 221, note 2.

12. Victor Mortet, par exemple, s'est appliqué à définir exactement les acceptions médiévales de termes d'architecture employés souvent sans discernement. On pense notamment aux articles du célèbre archiviste sur les mots « abside » (Bulletin Monumental, 1908, p. 162-166) ou «transept » (Bulletin Monumental, 1913, p. 265-2281) ou bien à la controverse sur le mot « deambulatorium » qui a opposé René de Lasteyrie et Victor Mortet dans des numéros de 1911 et 1912 de la même revue.

13. Voir par exemple : Philippe Bernardi, Métiers du bâtiment et techniques de construction à Aix-en-Provence à la fin de l'époque gothique, 1400-1550, Aix-en-Provence, 1995.

14. Sous la direction de Jean-Marie Pérouse de Montclos, CNRS, Paris, 1972. Le besoin d'un tel instrument de travail était vivement ressenti depuis longtemps : en 1912, Georges Durant écrivait dans le Bulletin Monumental : «Une des plus grosses difficultés dans l'archéologie du moyen âge réside, comme on le sait, dans le choix d'une terminologie expressive et, surtout, universelle. Depuis le début de la création de cette science, on n'est pas encore parvenu à s'entendre à ce sujet» (p. 201).

15. J.-A. Brutails, op. cit., p. 30.

16. Comme exemples récents d'analyse globalisante, faisant intervenir les entrées les plus diverses, de l'archéologie à la liturgie, voir : Der Dom zu Regensburg. Ausgrabung, Restauriegung, Forschung. Ausstellung anlässlich der Beendigung der Innenrestauriegung des Regensburger Domes, 1984-1988. Katalog der Ausstellung, Regensburg 14 Jul.-29 Nov. 1989, Munich-Zurich, 1989, ou bien Artistic Integration in Gothic Buildings, edited by Virginie Chieefo Raguin, Kathryn Brush, Peter Draper, University of Toronto, Toronto, Buffalo, London, 1995.

17. Les Nouvelles de l'archéologie, $n^{\circ}$ 53-54, automne-hiver 1993, "L'archéologie du bâti médiéval urbain ", dir. C. Arlaud, J. Burnouf, p. 5-69.

18. Les Nouvelles de l'archéologie, $n^{\circ}$ 53-54, automne-hiver 1993, p. 67-69. Voir, dans ce numéro, le dossier L'archéologie du bâti médiéval urbain, dir. C. Arlaud, J. Burnouf.

19. Archéologie du bâti, Journées d'études de Pont-à-Mousson, 20-23 septembre 1994, École du Patrimoine. Le point le plus récent sur la question a été publié par Catherine Arlaud, "L'archéologie du bâti », "Cent maisons médiévales en France (du XII ${ }^{\mathrm{e}}$ au milieu du XVI ${ }^{\mathrm{e}}$ 
siècle) ", dir. Y. Esquieu, J.-M. Pesez, monographie du CRA 20, Éditions du CNRS, Paris, 1998, p. 27-29, et par Florence Journot, "Archéologie du Bâti », La construction en pierre, Paris, éditions Errance, 1999, p. 133-163.

20. Les Nouvelles de l'archéologie, $n^{\circ} 57$ automne 1994. L'auteur, en accord avec C. Arlaud et J. Burnouf, faisait remonter cette pratique à plus d'une décennie. Elle a été suscitée, en effet, par la conjonction de deux mouvements : les transformations profondes qui ont altéré les centres-villes médiévaux -la chose n'est pas une nouveauté- et l'intérêt soulevé chez le grand public par ce que les démolitions mettaient sous ses yeux : un patrimoine méconnu, d'autant plus touchant qu'il évoquait puissamment la quotidienneté du Moyen Age. La rapidité qui caractérise, à notre époque, les transformations du tissu urbain, a fait de l'habitat médiéval et moderne un champ d'investigations privilégié et, l'urgence aidant, prioritaire. Les expériences menées dans le Vieux Lyon, qui a été, en 1964, l'un des premiers secteurs sauvegardés de France (loi Malraux 1962), ont été l'occasion de mettre au point, dans cette région, des méthodes spécifiques, exposées dans un cahier des Nouvelles de l'Archéologie (Les Nouvelles de l'Archéologie, n 53-54. Automne-hiver 93. Dossier: l'archéologie du bâti médiéval urbain, dir. C. Arlaud et J. Burnouf, p. 5-69). Voir Archéologie et projet urbain. De Luca editore, 1985.

21. La recherche a été largement développée en Italie. Voir le dossier présenté dans Archeologia dell'architettura. Supplemento ad Archeologia medievale XXIII, 1997 : Cagnana A. Ferrando I. : «L'esperienza scientifica dell'ISCUM e lo sviluppo dell'Archeologia dell'architettura in Liguria e in Lunigniana », p. 189-198 ;- Parenti R. : « Archeologia dell'architettura. Bilanco della situazione toscana », p. 175-180. Pour l'Espagne, dans le même numéro: Caballero Zoreda L. : "Analisis arqueologico de construcciones historicas en Espana. Estado de la cuestion », p. 147-158.

22. Voir le dossier présenté dans Archeologia dell'architettura. Supplemento ad Archeologia medievale XXIII, 1997 : Bonora F. : «Gli esordi dell'Archeologia del Costruito in Liguria : edifici abbandonati a Genova ", Archeologia dell'architettura. Supplemento ad Archeologia medievale XXIII, 1997, t. 2, p. 185-188;- De Minicis E.: «L'archeologia del costruito: esperienze in area laziale. Appendice di G. Voci », p. 167-172.

23. L'expression "archéologie d'élévation" a pu paraître plus justifiée dans le cadre de l'architecture monumentale, moins susceptible de modification récurrentes que la construction vernaculaire, et lorsque les élévations sont seules concernées. En effet, la différence essentielle avec la démarche précédente tient en l'absence de fouilles dans le sol et dans la non destruction de l'objet d'étude. Mais, dans la réalité, les choses sont plus mêlées. L'analyse des maçonneries peut s'accompagner a minima, et avec toutes les précautions requises, de légers sondages de vérification dans les joints; D. Kimpel (Bulletin Monumental, 1977, p. 196) a écrit, à propos de la mise en œuvre de la pierre, qu'« un tel système est reconnaissable à la vue, parfois l'on a besoin d'un couteau pour sonder ".

24. La littérature italienne sur le sujet est très riche. Voir, notamment: Archéologie et projet urbain. De Luca editore, 1985. Archeologia e Restauro dei Monumenti, a cura di R. Francovitch e R. Parenti, Firenze, 1988 :- Dal sito archeologico all'Archeologia del costruito. Conoscenza, Progetto e conservazione. Atti del convegno di studi, Bressanone, 3-6 Iuglio 1996. Padova, 1996, p. 39-48 ;- Della Torre S. Maria del Tiglio S. : « La 'archeologia sperimentale' nella tradizione del restauro architettonico », Archeologia dell'architettura. Supplemento ad Archeologia medievale XXIII, 1997, t. 2, p. 199-206.

25. Deshoulières, «Les premiers donjons de pierre dans le département du Cher ", Bulletin Monumental, 1948, p. 49-61.

26. L'application des méthodes de l'archéologie à un objet qui semblait devoir lui rester étranger (analyse matérielle du bâti et de sa "stratification " historique) est intervenue tôt. Dans un double article du Bollettino d'Arte de 1915 sur l'église de Sainte-Marie-Majeure, Mgr Giovanni Biasiotti présentait les résultats de sa recherche qui l'avait amené à réviser les hypothèses fallacieuses sur la datation et l'identification de l'édifice; il appuyait ses conclusions sur des 
études du bâti et des sondages dans les maçonneries: reprises, bouleversements localisés, épaisseur des lits de mortiers, diversité des dimensions des briques, diversité dans la coloration des briques, traces de grattage indiquant un remploi, cassures et refaçonnage des matériaux, ... Gionanni Biasiotti : "La basilica di S. Maria Maggiore a Roma ", Bollettino d'Arte, 1915, fasc. 1, p. 20-32, et fasc. 5, p. 136-148.

27. Dans le cadre de restaurations, les interventions dans le bâti peuvent favoriser la fouille. Ainsi à Lyon, le démontage d'une partie du décor sculpté et des contreforts de la Vieille Manécanterie, en 1981, a permis de situer la place de l'opus quadratum dans la chronologie du bâtiment, d'origine carolingienne. Voir: Madeleine Vialettes : «Les bâtiments de la »Vieille Manécanterie « de la cathédrale Saint-Jean de Lyon », Bulletin Monumental, 1995, 153-I, p. 47-63.

28. On fouille effectivement un mur, lorsqu'on est amené à en démonter des composantes ou à suivre sa démolition.

29. Voir la récente synthèse qui fait le point sur les différentes formes de relevés architecturaux : Les techniques du relevé d'élévation, Table ronde organisée par le CÉRIAH et la Bibliothèque municipale de Lyon, 27 mai 1997, textes réunis par A. Baud et I. Parron, Centre International d'Études Romanes, Tournus, 1998, p. 145-188.

30. Dans ce domaine, la précision du vocabulaire archéologique est encore faible et l'identification scientifique des types reste à faire.

31. L'échafaudage dans le chantier médiéval, ouvrage collectif, Documents d'Archéologie en Rhône-Alpes, Lyon, 1996. Pour l'analyse d'un cas concret, voir: N. Reveyron : «Technologie médiévale et archéologie d'élévation : l'échafaudement du chevet de la cathédrale Saint-Jean de Lyon (dernier quart XII ${ }^{\mathrm{e}}$ - premier tiers XIII ${ }^{\mathrm{e}}$ )», Archéologie Médiévale, 1998, p. 91-129.

32. Voir par exemple: K. Friedrich, Die Steinbearbeitung in ihrer Entwiklung von 11. zum 18. Jahrhundert, Augsburg, 1932 ; E. Nagy: «La datation des pierres sculptées d'après les traces d'outils ", La formation et le développement des métiers au Moyen Age (VI ${ }^{\mathrm{e}}$-XIV ${ }^{\mathrm{e}}$ siècles). Colloque International de Budapest, 1973, Budapest, 1977, p. 107-119 ; Arnaud Timbert, « Usage du marteau taillant bretté à la période gothique ", Comment construisait-on au Moyen Age?, Dossiers d'Archéologie, n² 251, mars 2000, p. 76-77.

33. Sur le sujet, la littérature est très abondante. Les efforts de théorisation ont été considérables depuis les années 50. Voir notamment: E. Nicolas, «Les signes lapidaires: approche méthodologique", Pierre et métal dans le bâtiment au Moyen Age, Etudes réunies par 0. Chapelot et P. Benoit. EHESS, Paris, 1985, p. 185-195 ; J-L Van Belle, « Les marques de tailleurs de pierre. Pour une problématique régionale et internationale ", Artistes, artisans et production artistique au Moyen Age, Colloque international CNRS Dir. X. Barral i Altet, Paris, 1986, p. 519-524; idem, «Les marques de tailleurs de pierre. Remarques sémiologiques », Actes du Colloque International de Glyptographie de Pontevedra, juillet 1986, Braine-le-Château et Pontevedra, 1986, vol.1, p. 247-258; N. Reveyron, "Herméneutique du signe. Approche épistémologique, conditions et limites d'une recherche ", Actes du $\mathrm{XI}^{\mathrm{e}}$ Colloque International de Glyptographie, Palma de Majorque, juillet 1998, Bruxelles, 2000, p. 337-357.

34. Dans ce domaine encore, les travaux les plus récents, par exemple en Alsace, en île-de-France et singulièrement en Belgique où la recherche glyptographique est très développée, ont amené des découvertes majeures. Pour un rapide état de la question, voir : N. Reveyron, « Remarques sur les fonctions des marques lapidaires dans le chantier médiéval», Bulletin du Centre International d'Études Romanes, Tournus (à paraître, 2001).

35. Par exemple, D. Kimpel, «Le développement de la taille en série et son rôle dans l'histoire économique ", Bulletin Monumental, 1977, p. 195-222 ; D. Prigent, «Etude statistique d'appareil à l'intérieur de l'abbaye de Fontevraud. Aspects méthodologiques», Revue Archéologique de l'Ouest, 6, 1989, p. 155-172; A. Salamagne, «Recherches épistémologiques sur l'architecture médiévale : l'exemple de la porte de Notre-Dame de Douai (1454-1457). I-Prolégomènes », 117e 
congrès national des sociétés savantes. Clermont-Ferrand, 1992, 2e coll. Carrières et constructions, p. 73-87.

36. D. Prigent, « Méthodes d'investigations archéologiques utilisées à l'abbaye de Fontevraud ", Fontevraud, histoire et archéologie, № 4-comité d'histoire fontevriste, 1995-1996, p. 17-43.

37. Pour une vue d'ensemble de ces problématiques, voir : Au fil du chantier - Archéologie de la construction au Moyen Age, Catalogue de l'exposition, Lyon, 1997.

38. L'étude des irrégularités de plan remonte aux premières investigations des « antiquaires » du $\mathrm{XIX}^{\mathrm{e}}$ siècle; le stade de l'interprétation symboliste a été vite dépassé au profit de l'analyse archéologique. Voir l'article ancien de R. de Lasteyrie (« La déviation de l'axe des églises est-elle symbolique?», Bulletin Monumental, 1905, p. 422-459) qui fait le point, de façon polémique, sur le sujet au début du siècle.

39. J-C. Biaudet, W. Stöckli, H. Meylan, Ph. Jaton, M. Grandjean, Cl. Lapaire, La cathédrale de Lausanne, Berne, 1975.

40. R. Branner, La cathédrale de Bourges, Paris et Bourges, 1962 ; Marc Férauge, Pierre Mignerey : "L'utilisation du fer dans l'architecture gothique: l'exemple de la cathédrale de Bourges ", Bulletin Monumental, 1996, p. 129-148.

41. N. Reveyron, «Les échanges artistiques entre Vienne et Lyon. Inventions et influences dans l'architecture romane des cathédrales Saint-Maurice et Saint-Jean ", Bulletin de la Société des Amis de Vienne, numéro spécial, juin 1994, p. 81-97.

42. Dans le sud-est, deux édifices -les Célestins d'Avignon et la cathédrale de Carpentras- ont encore pour façade le mur de clôture occidental. L'archéologie du sous-sol en retrouve parfois des traces. G. Démians d'Archimbaud interprète comme une clôture de chantier le mur nord-sud établi au milieu de la nef de la cathédrale de Digne à la fin du XII ${ }^{\mathrm{e}}$ siècle et destiné à délimiter un pôle occidental de travail. Voir : Démians d'Archimbaud G. : « De l'an Mil à l'incendie du XII siècle » et « La nouvelle cathédrale au XIII" siècle : le chantier de construction », Notre-Dame du Bourg, une vie de cathédrale, catalogue de l'exposition, Digne, 1990, p. 27-29 et 32-34.

43. Il arrive que la reconstruction d'une église la prive momentanément d'une de ses attributions: ainsi, à Lausanne, les reliques de la Vierge ont-elles connu un «exil » d'une cinquantaine d'années. La cathédrale de Lausanne. 1975, op. cit., p. 45 sq.

44. Les rapports de l'édifice avec les espaces de circulation apparaissent dans l'organisation des portes et son devenir. Voir, notamment: J. Mallet «Le type d'église à passage en Anjou. Essai d'interprétation », Cahier de Civilisation Médiévale, 1982, p. 49-62.

J. Passini: Villes médiévales du chemin de Saint-Jacques de Compostelle (de Pampelune à Burgos). Editions Recherches sur les Civilisations, n 47, Paris, 1984.

45. Le sol et l'immeuble, EFR-PUL, 1995 ;- Cent maisons médiévales en France. Un corpus et une esquuisse, dir. Y. Esquieu, J.-M. Pesez, Paris, CNRS, 1998. Pour une vue d'ensemble sur la question: Y. Esquieu : «La maison médiévale urbaine en France. Etat de la recherche », Bulletin Monumental, 1995, p. 109-142.

46. Y. Esquieu, «La maison médiévale dans les agglomérations en Provence et dans le sillon rhodanien ", École antique de Nîmes, bulletin n 24, 1993-98, p. 83-96 ;- V. Lassalle, « Le décor des façades de quelques maisons romanes du Gard », ibidem, p. 97-115.

47. M.-G. Colin, I. Darnas, N. Pousthomis-Dalle, L. Schneider, La maison du castrum de la bordure méridionale du Massif Central, Archéologie du Midi Médiéval, supplément 1, 1996, p. 159.

48. Il s'agissait de créments, c'est-à-dire de terrains délaissés par la Saône et relevant, de ce fait, de la puissance publique

49. N. Reveyron, La cathédrale Saint-Jean Baptiste, Lyon. Lyon, 1997, p. 16-17.

50. Laboratoire des sciences de l'habitat, groupe «Lumière et rayonnement électromagnétique ». Dir. Marc Fontoynont. L'étude a été réalisée par Luc Seiwert. Voir: Luc Seiwert, Étude de l'éclairage naturel de la cathédrale Saint-Jean de Lyon. Maîtrise de T. P. E., dir. V. Berrutto. Dactylographiée, mai 1998. 
51. N. Reveyron, «Lumières gothiques. Évolution du voûtement et de l'éclairement dans la cathédrale de Lyon au XIII ${ }^{\mathrm{e}}$ siècle ", Pierre, lumière, couleurs. Hommage offert à Anne Prache, textes réunis par F. Joubert et D. Sandron. PUPS, Paris, 1999, p. 165-184. Notons que l'étude de l'éclairement dans son état de la fin $\mathrm{du} \mathrm{XX}^{\mathrm{e}}$ siècle et la modélisation par ordinateur de l'évolution historique de l'éclairement de la cathédrale a permis non seulement de donner les jalons d'une «histoire lumineuse » de l'édifice, mais de voir aussi se dessiner une histoire des incidences lumineuses venues du contexte urbain sur l'édifice.

INDEX

Keywords : on line, electronic journal, ejournal, heritage, history of art, France, archaeology, architecture

Mots-clés : inventaire général, en ligne, journal, revue électronique, revue numérique, périodique, patrimoine, histoire de l'art, France, archéologie, architecture

\section{AUTEUR}

\section{NICOLAS REVEYRON}

Professeur d'histoire de l'art et archéologie du Moyen Age, Université de Lyon 2 18, quai ClaudeBernard 69365 Lyon Cedex 7. nicolas.reveyron@univ-lyon2.fr 